Article

\title{
An Electrochemical and Raman Scattering Dual Detection Biosensor for Rapid Screening and Biomolecular Profiling of Cancer Biomarkers
}

\author{
Shuvashis Dey ${ }^{1} \mathbb{D}$, Emtiaz Ahmed ${ }^{1}$, Pranjal Satishchandra Somvanshi ${ }^{1}$, Abu Ali Ibn Sina ${ }^{1, * \mathbb{D}}$, \\ Alain Wuethrich $1, * \mathbb{D}$ and Matt Trau 1,2,*D \\ 1 Centre for Personalized Nanomedicine, Australian Institute for Bioengineering and Nanotechnology (AIBN), \\ The University of Queensland, Brisbane, QLD 4072, Australia; s.dey@uq.edu.au (S.D.); \\ e.ahmed@uq.edu.au (E.A.); p.somvanshi@uq.net.au (P.S.S.) \\ 2 School of Chemistry and Molecular Biosciences, The University of Queensland, Brisbane, QLD 4072, Australia \\ * Correspondence: a.sina@uq.edu.au (A.A.I.S.); a.wuethrich@uq.edu.au (A.W.); m.trau@uq.edu.au (M.T.)
}

Citation: Dey, S.; Ahmed, E.; Somvanshi, P.S.; Sina, A.A.I.

Wuethrich, A.; Trau, M. An

Electrochemical and Raman Scattering Dual Detection Biosensor for Rapid Screening and Biomolecular Profiling of Cancer

Biomarkers. Chemosensors 2022, 10, 93. https://doi.org/10.3390/

chemosensors 10030093

Academic Editors: Christian Dusny and Alexander Grünberger

Received: 31 January 2022

Accepted: 25 February 2022

Published: 28 February 2022

Publisher's Note: MDPI stays neutral with regard to jurisdictional claims in published maps and institutional affiliations.

Copyright: (C) 2022 by the authors. Licensee MDPI, Basel, Switzerland. This article is an open access article distributed under the terms and conditions of the Creative Commons Attribution (CC BY) license (https:// creativecommons.org/licenses/by/ $4.0 /)$.

\begin{abstract}
Detecting circulating biomarkers sensitively and quantitatively is paramount for cancer screening, diagnosis, and treatment selection. Particularly, screening of a panel of circulating protein biomarkers followed by mapping of individual biomarkers could assist better diagnosis and understanding of the cancer progression mechanisms. Herein, we present a miniaturized biosensing platform with dual readout schemes (electrochemical and Surface enhanced Raman scattering (SERS)) for rapid cancer screening and specific biomarker expressional profiling to support cancer management. Our approach utilizes a controlled nanomixing phenomena under alternative current electrohydrodynamic condition to improve the isolation of cancer-associated circulating proteins (i.e., Epidermal growth factor receptor (EGFR), BRAF, Programmed death-ligand 1 (PD-L1)) with antibody functionalized sensor surface for rapid and efficient isolation of the targets and subsequent labelling with SERS nanotags. The method employs Differential Pulse Voltammetry (DPV) for rapidly screening for the presence of the circulating proteins on biosensor surface irrespective of their type. Upon positive DPV detection, SERS is applied for sensitive read-out of individual biomarkers biomarker levels. In a proof-of-concept study, we demonstrate the dual detection biosensor for analysing circulating BRAF, EGFR and PDL-1 proteins and successfully screened both ensemble and individual biomarker expressional levels as low as $10 \mathrm{pg}(1 \mathrm{ng} / \mathrm{mL})$. Our findings clearly indicate the potential of the proposed method for cancer biomarker analysis which may drive the translation of this dual sensing concept in clinical settings.
\end{abstract}

Keywords: Surface-enhanced Raman scattering; cancer biomarkers; differential pulse voltammetry; dual detection mode; liquid biopsy

\section{Introduction}

Cancer is a dynamic and heterogenous disease with treatment relying on accurate analysis of biomarkers in cancer tissue and liquid biopsy [1,2]. However, the analysis of tissue biopsy might fail to reflect cancer heterogeneity and only provides a tumour snapshot at the time of sampling. Due to these shortcomings the analysis of biomarkers in liquid biopsy has been regarded increasingly as a less-invasive diagnostic to infer tumour state, treatment response, and tumour-immune evasion [3-6]. Among the different types of biomarkers in circulation, cancer cell secreted proteins (i.e., soluble proteins) have been shown to be particularly useful for diagnostics, where the level of circulating protein biomarkers can serve as proxy for expression in primary tumour [7,8]. However, the analysis of soluble proteins is difficult because the biomarker is diluted significantly in circulation and its specific detection complicated due to abundance of non-target molecules. 
Over the year, a number of technologies have been reported for the analysis of soluble proteins including fluorescence measurement, colorimetric tests, enzyme-linked immunosorbentoassay (ELISA), etc. [9-15]. Among the assay detection formats, electrochemistry offers simplicity, speed, sensitivity, and portability which are key features to develop clinically translatable diagnostics $[16,17]$. Electrochemical biosensors typically have three components including biorecognition element, signal transducer and an electrode array for electrochemical setup [18]. In such setup, the change in electrochemical signal during the electrochemical reaction due to the presence of captured biomolecules on the sensing surface are recorded and analysed to detect the biomolecules in a sample. For example, Ilkhani et al., developed a differential pulse voltammetry (DPV) biosensor to detect circulating EGFR on anti-EGFR functionalized device with a limit of detection of as low as 50 pg/mL [19]. Moon et al., developed a immunosensor patterned with Au nanowire array on sensing surface for efficient anti-PSA antibody immobilization and successfully detected prostate specific antigens (PSA) at femtogram level [20]. Bravo et al., developed a polyvinyl alcohol coated silver nanoparticle patterned immunosensor for specific and sensitive detection of epithelial cell adhesion molecules (EpCAM) [21]. More recently, Moazzam et al., reported an electrochemical sensor for PDL-1 protein detection with ultrasensitivity [22]. The method employs capture antibody immobilized gold coated magnetic nanoparticels and HRP labelled detection antibodies for PDL-1 capture and electrochemical signal detection. Using this scheme, they have successfully detected PDL-1 as low as 15 attomolar from undiluted blood. In another study, Dey et al., reported a microfabricated electrochemical sensor for rapid and sensitive detection of BRAF proteins from cell lysate [23].

In recent years, SERS based methods gain significant attention for biomolecular analysis due to multiple factors including- unique and strong fingerprint signals from SERS nanotags, stability of the nanotags, multiplexibility (i.e., up to 32 reporters) [24,25]. In principle, when a laser shines on nanoparticles immobilized with Raman reporter molecules, it initiates local hotspots and enhances the Raman signal intensities of the reporter molecules by multiple orders of magnitude. Until now, there are number of published research works that demonstrate the potential of SERS based methods for multiplex biomarker detection with high sensitivity [26,27]. For example, Wang et al., integrated SERS in a miniaturized platform for detecting cancer specific soluble protein (HER2) as low as $10 \mathrm{fg} / \mathrm{mL}$ [28]. More recently Li et al., reported a SERS nanopillar array system for multiplexed analysis of cytokines and analysed patient samples targeting four clinically relevant cytokines down to attomolar level [29]. Along with nanoparticle-based approaches for SERS assays, direct utilization of nanopatterned surfaces are under investigation and reported in a number of studies for their significant potential of being a label free SERS platform for biomolecule analysis. For example, Yang et al., reported a hybrid SERS substrate containing hierarchical micro/nano structures with superhydrophobic/hydrophobic characteristics for rapid and highly sensitive SERS detection of biomolecules as low as femtomolar level. Using this highly structured SERS platform they have successfully detected rhodamine 6G as low as $10^{-14} \mathrm{M}$ using only $5 \mu \mathrm{L}$ sample [30]. Similarly, Xu et al., developed a flexible transparent SERS substrate for the detection of trace chemicals such as pesticides. In this approach, femtosecond laser induced plasma assisted ablation (LIPAA) technique was applied to pattern gold or silver nanoparticles on flexible transparent fluorinated ethylene propylene (FEP) film to achieve a SERS substrate. The potential of this platform (e.g., Ag NPs/FEP) was demonstrated the detection of pesticide residues in apples with a detection limit of $0.1 \mathrm{mg} / \mathrm{Kg}$ for thiram [31]. In another study, Kozhina et al., reported a template assisted synthesis method for pattering silver nanowires (NWs) on a substrate with dendritic nanostructures on their tips. The application of iodide-containing electrolyte on tracking membranes (TMs) enables to synthesize dendritic nanostructures on the vertices of silver NWs. Furthermore, they have modelled the electromagnetic field near the patterned nanostructures upon application of visible laser radiation and reported considerable electric filed enhancement [32]. Kozhina et al., also developed a simple method for patterning metal nanowires (NWs) by utilizing polymer track-etched membranes (TM) as template for 
electrodeposition. Using this method, they developed self-assembled bundles of Ag-NWs where adjacent nanowires formed local hot spots. They have extensively studied the effect of NW lengths on SERS intensities and found that $1.6 \mu \mathrm{m}$ is optimal length for obtaining maximum SERS spectra intensities. Furthermore, they have also found that the SERS signal intensities remain $49 \%$ higher when the analyte (4-Mercaptophenylboronic acid) was added to the wet surface then the dry surface [33].

Despite the significant advancement in technology development and their promising results for circulating biomarker analysis, the simultaneous isolation of multiple clinically relevant protein targets followed by their ensemble and individual expressional analysis has not been attempted. We rationalised that combining electrochemical and SERS readouts into a dual-detection biosensor could provide a strategy for rapid cancer screening and subsequent molecular profiling. Electrochemistry provides fast read-outs and is suitable for the point-of-care setting, which are highly desirable features for cancer screening. The electrochemical ensemble measurement screens for the presence of a panel of circulating biomarkers. If a positive screening result is obtained, subsequent analysis of the individual circulating biomarkers by highly sensitive and multiplex SERS read-out delivers a molecular profile to guide cancer management.

In this proof-of-concept study, we report a dual detection biosensor platform that screens for a panel of cancer-associated protein biomarkers (i.e., BRAF, EGFR, PDL-1) by an electrochemical ensemble measurement using DPV and profiles the individual biomarker levels using SERS. Within this biosensor platform, the application of alternating current electrohydrodynamics (ac-EHD) induces a nanomixng phenomenon that facilitates rapid and enhanced target capture by increasing the collision frequencies between target molecules and capture antibodies, reduces nonspecific adsorption and facilitates labelling of antibody coated SERS tags to the captured biomolecules $[23,28,34]$. Following capture, the biosensor platform utilizes an DPV for ensemble biomarker detection that can sense minute amount of molecule absorbance on the sensing surface and present results in the form of peak current change. To further investigate the presence of individual biomarkers on sensing surface and their level of expression, we adopted a SERS method by engaging laser to the sensing surface to record SERS signals that correspond to the amount of individual biomarkers captured on the surface. Our method is simple, rapid (total 10 min assay time), sensitive and provide two independent readouts from the same sample to extract crucial molecular information of the disease that may also help to increase the confidence level of the biosensing technique. We believe this screening technique for assaying ensemble and individual clinically relevant cancer specific soluble biomarkers can be utilized for conveying critical information for disease management and may have the potential to be integrated in the clinical settings.

\section{Materials and Methods}

All the reagents including pure proteins, antibodies and other chemicals were purchased from BioLegend (Wangara, Western Australia, Australia), R\&D Systems (Noble Park North, Victoria, Australia) and Sigma Aldrich (Sydney, New South Wales, Australia). Device fabrication was performed at the Australian National Fabrication Facility, Queensland Node.

\subsection{Device Design and Fabrication}

The first step of device fabrication was started with device design using L-edit software V15 (Tanner Research, Wilsonville, OR, USA) and inscribing the design on a photomask using direct laser write. The device design contains a central circular electrode surrounded by a ring electrode. Both of the electrode designs are connected with a pair of connector pads for external electric power. After mask development, glass wafers (4 inch diameter, $1 \mathrm{~mm}$ thick) were spin coated with a negative photoresist (AzNOLF 2020), soft baked at $110{ }^{\circ} \mathrm{C}$ temperature for $2 \mathrm{~min}$ before being UV exposed with the photomask containing the desired design. This step was followed by a hard bake step for another 2 min at $110{ }^{\circ} \mathrm{C}$ before 
the wafers were developed with developing solution (Az726) to generate a photoresist patterned wafer. Then the wafers were transferred into E-beam chamber for gold deposition. Finally, after lift off with acetone, wafers were diced to generate individual devices. The detailed scheme of the fabrication process in presented in Supporting Information (SI) Figure S1.

\subsection{SERS Nanoparticle Synthesis}

The gold nanoparticles were synthesised following a standard protocol of citrate reduction of $\mathrm{HAuCl}_{4}$ to yield spherical nanoparticles of $\sim 60 \mathrm{~nm}$ diameter as shown by the SEM image in SI Figure S2 [35]. Next, $10 \mu \mathrm{L}$ of $1 \mathrm{mM}$ Raman reporters dissolved in ethanol (either 4-mercaptobenzoic acid (MBA), 5,5'-Dithiobis-(2-Nitrobenzoic Acid) (DTNB) and 2,3,5,6-tetrafluoro-4-mercaptobenzoic acid (TFMBA)) were added to $1 \mathrm{~mL}$ of nanoparticle solution. Just after this step, $2 \mu \mathrm{L}$ of $1 \mathrm{mM}$ DSP (dithiobis (succinimidyl propionate)) (Thermo Fisher Scientific) in dimethyl sulfoxide was added to each tube and left them for overnight incubation at room temperature. Following incubation, unreacted chemicals were removed by centrifuging the solution at $7600 \mathrm{rpm}$ for $10 \mathrm{~min}$ and resuspending the palette in $200 \mu \mathrm{L}$ of $0.1 \mathrm{mM}$ PBS (Phosphate-buffered saline). After this step, $2 \mu \mathrm{g}$ of anti-EGFR, anti-BRAF and anti-PDL-1 were added to DTNB, MBA and TFMBA solutions, respectively. After $30 \mathrm{~min}$ incubation at room temperature, the solutions were centrifuged at $600 \mathrm{~g}$ for another $10 \mathrm{~min}$ and palettes were resuspended in $200 \mu \mathrm{L}$ of $0.1 \%(w / v)$ BSA for $0.5 \mathrm{~h}$. After this, individual tubes were centrifuged at $600 \mathrm{~g}$ for another $10 \mathrm{~min}$ and palettes were resuspended in $200 \mu \mathrm{L}$ of $1 \mathrm{mM}$ PBS solution. Following SERS tag preparation, $60 \mu \mathrm{L}$ of each SERS nanotag solutions were transferred to respective cuvettes and energized with Raman laser to confirm SERS signals from reporter molecules after functionalization (SI Figure S3). Then, $50 \mu \mathrm{L}$ of each nanotag solution was mixed into a separate tube for making SERS tag cocktail for multiplexed detection. Finally, the newly prepared SERS nanotags were stored at $4{ }^{\circ} \mathrm{C}$ freeze and used subsequently in the experiments.

\subsection{Assay Protocol, Signal Detection and Analysis}

Prior to start functionalization, an antibody mix solution (stock) was prepared by transferring and mixing $50 \mu \mathrm{L}$ of individual antibody solutions $(1 \mathrm{ng} / \mathrm{mL})$ ) into an Eppendorff tube. For each device functionalization, DSP solution was added to the chip surface and incubated for $2 \mathrm{~h}$. Then, $10 \mu \mathrm{L}$ of antibody mixed solution (stock) was added to each device surfaces for $2 \mathrm{~h}$ to immobilize one type of antibody to each electrode and then washed with $1 \mathrm{mM}$ PBS for 3 times to remove unbound molecules. Then, the functionalized surfaces were rinsed with $10 \mathrm{mM}$ (pH 8.0) Tris- $\mathrm{HCl}$ buffer to neutralized unreacted DSP molecules [36]. Finally, 0.1 $\mu \mathrm{M}$ 6-mercapto-1-hexanol (Sigma Aldrich, Australia) was incubated on the device surfaces for 10 min to block the remaining surfaces.

For target capture experiments, designated amount of target protein(s) solution $(10 \mu \mathrm{L})$ was added to the device surface and nanomixing was initiated by applying an ac electric field $\left(f=500 \mathrm{~Hz}, \mathrm{~V}_{p p}=800 \mathrm{mV}\right.$ ) for $5 \mathrm{~min}$ to facilitate collision between target proteins and antibodies. After capture experiments, PBS wash was performed three times to remove non-specifically adsorbed molecules. Then labelling experiments were conducted by adding $10 \mu \mathrm{L}$ of SERS tag cocktail to the device under ac-EHD for $5 \mathrm{~min}$ and washed with $1 \mathrm{mM}$ PBS again. For DPV measurement of the electrodes in the device, $10 \mu \mathrm{L}$ of ferri/ferrocyanide redox couple (i.e., $2.5 \mathrm{mM}\left[\mathrm{Fe}(\mathrm{CN})_{6}\right]^{3-} /\left[\mathrm{Fe}(\mathrm{CN})_{6}\right]^{4-}(1: 1)$ and $0.1 \mathrm{M} \mathrm{KCl}$ in $10 \mathrm{mM}$ phosphate buffered saline at $\mathrm{pH}$ 7.4) solution was added to the electrode surface and current was recorded with a CHI650D electrochemical workstation (CH Instruments, Bee Cave, TX, USA). The DPV measurements were recorded from -1.0-1.0 V with a pulse amplitude of $50 \mathrm{mV}$, a pulse width of $50 \mathrm{~ms}$, a potential step of $5 \mathrm{mV}$, and a pulse period of $100 \mathrm{~ms}$. The DPV currents recorded before target capture and after captured molecule 
labelling with SERS tags (i.e., captue + labelling) were utilized to calculate the relative current change $\left(\% \mathrm{i}_{\mathrm{r}}\right)$. The formula is as follows:

$$
\% \mathrm{i}_{\mathrm{r}}=\left[\left(\mathrm{i}_{\text {before capture }}-\mathrm{i}_{\text {after labelling }}\right) / \mathrm{i}_{\text {before capture }}\right] \times 100 \%
$$

For SERS data recording, microdevices with SERS-tagged protein targets were transferred to WITec Alpha300 R microspectrometer and energized with $632 \mathrm{~nm}$ laser source. For individual SERS scan, an area of $60 \mu \mathrm{m} \times 60 \mu \mathrm{m}$ (60 pixels $\times 60$ pixels) was selected and signals were recorded with $50 \mathrm{~ms}$ integration time. For each multiplexed analysis, average SERS spectra were calculated from three different spots on the same device.

\section{Results}

\subsection{Working Scheme}

The workflow of the dual detection biosensor platform is shown in Figure 1. In brief, the microfabricated dual detection biosensor platform comprises of an inner circular electrode and outer ring electrodes which can be connected to an external electric power source for ac-EHD induced nanomixing and electrochemical sensing. For specific capture of target molecules, the dual detection biosensor surface was immobilized with a cocktail of capture antibodies (i.e., anti-EGFR, anti-BRAF, and anti-PDL-1) utilizing a DSP linker as described in the experimental section. DSP has disulphide bond that forms a strong bond with the gold surface of the biosensor in one side and also have amine reactive groups that reacts with the antibodies to form a stable immobilization of antibodies on the biosensor surface. Each step of successful immobilization of molecules were confirmed by the relative DPV current changes as presented in SI Figure S4. Following immobilization, spiked sample containing individual protein biomarker (i.e., EGFR, BRAF and PDL-1) or a mixture of three protein biomarkers was added to the biosensor's capture zone and connected with ac signal generator for inducing nanoscopic mixing for enhance biomarker capture and remove nonspecific non-target adsorption. For labelling with secondary antibody coated SERS nanotags, a solution containing the mixture of all the three antibody functionalized SERS nanotags (MBA-BRAF, DTNB-EGFR, TFMBA-PDL-1) was added to the surface under nanomixing condition for labelling each captured protein with their corresponding SERS nanotag. Critical to the sensitive protein biomarker detection was the Raman reporter signal enhancement of the SERS nanotags through electromagnetic and chemical effects. The SERS nanotags were based on $\sim 60 \mathrm{~nm}$ gold nanoparticles conjugated with Raman reporters and this pairing has been shown to provide Raman signal enhancements of $>10^{5}$ and signal uniformity [37-39].

For electrochemical experiments, we monitored the decrease of DPV current during each step of functionalization and after target biomolecule capture on the sensing surface. This decrease in the current is due to increased biomolecule adsorption on the surface which hinders the redox reaction of the $\left[\mathrm{Fe}(\mathrm{CN})_{6}\right]^{3-/ 4-}$ redox system. The difference in Faradic current signal correspond to the target biomolecule captured on the sensor surface. For SERS mapping, the sensor surface with captured biomolecules labelled with SERS tags was scanned under the Raman microscope and signals were recorded that reflect the presence of target protein biomarkers on the sensing area (characteristic peaks for MBA, DTNB and TFMBA are $1080 \mathrm{~cm}^{-1}, 1335 \mathrm{~cm}^{-1}$ and $1376 \mathrm{~cm}^{-1}$ ). 


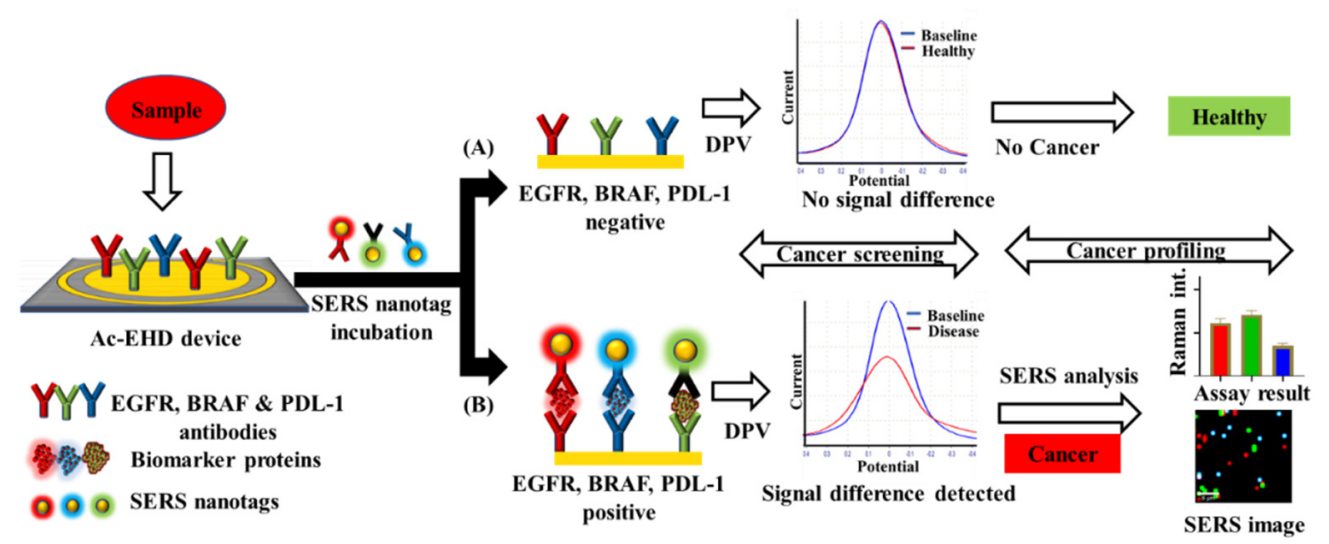

Figure 1. Schematic of the dual detection biosensor platform. The sample was added to the antiEGFR, BRAF, and PDL-1 antibody functionalised biosensor. Screening of samples that are negative for circulating EGFR, BRAF, and PDL-1 follows scheme (A) where DPV signals remain similar before and after sample analysis. If the sample contains any of the three circulating proteins, the assay follows (B). The dual detection biosensor initially detects the presence the protein panel by a detectable DPV signal change. Subsequently, SERS mapping of the biomarker captured and SERS tag labelled surface is performed to read-out the individual protein expressions levels.

\subsection{Specificity for the Ensemble and Individual Detection of Circulating EGFR, PDL-1, and BRAF}

Detection specificity is one of the major parameters to evaluate the merit of a biosensor. To evaluate capability of our biosensor to specifically capture target EGFR, BRAF, and PDL-1, we challenged the platform with a series of control experiments. We initially ran a control experiment with PBS and measured the current change in the DPV experiment. From the results in Figure 2a, negligible change of current which indicates the stability of our dual detection biosensor platform. Further to investigate the specificity of capture antibodies for the target proteins, we ran a set of experiments with non-target MCSP protein and did not encounter any significant changes in the Faradic current, which demonstrated the high detection specificity. The application of ac-EHD could be attributed for the negligible nonspecific adsorption as it creates nanoscopic force on the biosensor surface and facilitates clearing of the weakly bonded molecules [34]. Furthermore, we challenged our antibody functionalized biosensor with solutions of secondary antibody labelled SERS nanotags and did not observe significant current reductions. All these results clearly indicate very low or no nonspecific adsorption on the sensor surfaces, a very desirable quality of a biosensor. We then performed separate experiments with $100 \mathrm{pg}(10 \mathrm{ng} / \mathrm{mL})$ of each target proteins and recorded the DPV current change. The binding of target protein resulted in a significant current reduction $\left(\% \mathrm{i}_{\mathrm{r}} \sim 80 \%\right)$ which clearly demonstrates the capability of our biosensor for detecting individual protein of interest (Figure 2b). We also performed Raman scanning on the platforms for detecting SERS signals from the corresponding SERS nanotags to evaluate the essence of SERS signals specificity. It can be clearly seen from the Figure $2 c$ that SERS signal was significantly strong for experiments where experiments were performed with $100 \mathrm{pg}$ EGFR protein solution $(10 \mathrm{ng} / \mathrm{mL})$ for capture and then labelled with DTNB-EGFR SERS nanotags than the control experiment performed without target protein (i.e., no EGFR protein capture). This result clearly demonstrates the specificity of DTNB-EGFR SERS nanotags for target protein. Similar experiments were also performed for demonstrating the specificity of MBA-BRAF and TFMBA-PDL-1 which are presented in Figure 2d,e. 
(a)

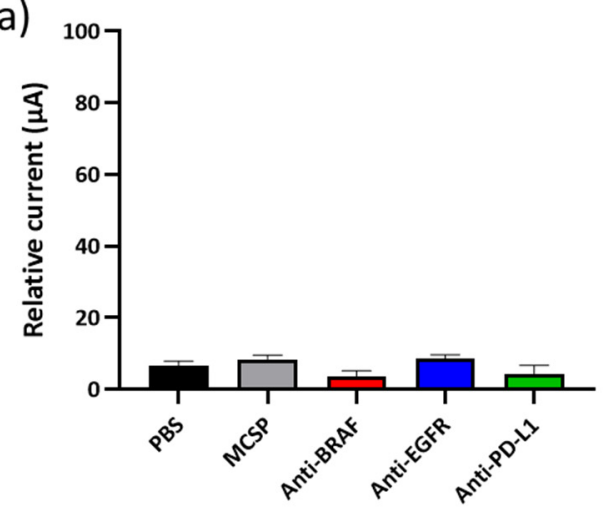

(c)

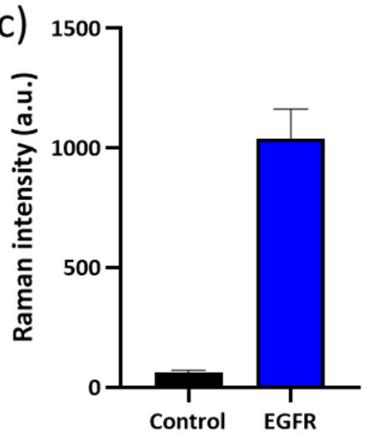

(d)

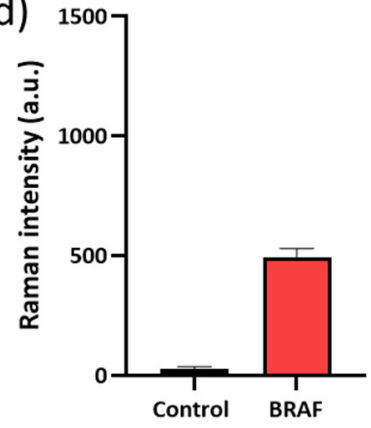

(b)

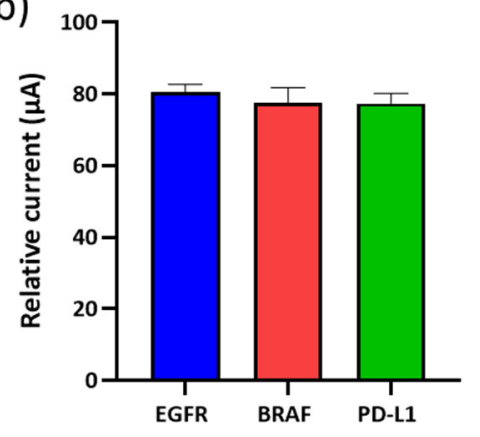

Figure 2. Detection specificity of dual detection biosensor platform. (a) presents the relative adsorption data for different control experiment where the current reduction was negligible $(>10 \%)$. (b) shows the observed DPV current reduction for $100 \mathrm{pg}(10 \mathrm{ng} / \mathrm{mL})$ target protein capture. The error bars represent the standard deviation from three replicate runs. SERS intensities for positive detection of EGFR (100 pg) and its control intensity (i.e., no EGFR protein) is presented in (c). (d,e) represents the SERS intensities of positive experiments with BRAF and PDL-1 proteins (100 pg) in respect to their control experiments (i.e., no targets), respectively. $(n=3)$ The error bars represent the standard error from three replicate runs.

\subsection{Sensitivity for Individual Detection of Circulating EGFR, BRAF and PDL-1}

To evaluate the feasibility of this platform for ensemble and individual circulating protein detection, it is important to individually challenge the functionalized surface with different concentration of target proteins separately and then assay corresponding electrochemical and SERS signals. Firstly, we ran experiments with three different amounts of EGFR samples, i.e., $10 \mathrm{pg}(1 \mathrm{ng} / \mathrm{mL}), 50 \mathrm{pg}(5 \mathrm{ng} / \mathrm{mL})$ and $100 \mathrm{pg}(10 \mathrm{ng} / \mathrm{mL})$ and recorded both DPV and SERS signals (Figure $3 a, d$ ). From the electrochemical assay small signal reduction was recorded for $10 \mathrm{pg}$ proteins $(\sim 30 \%)$ where DPV signal was significantly reduced when the experiment was performed with 100 pg EGFR proteins $(\sim 80 \%)$. A possible explanation of these observation could be attributed to the larger surface coverage in case of $100 \mathrm{pg}$ protein experiments that inhibited the diffusion and the electron transfer towards the electrode surfaces (i.e., hinder redox reaction) hence produced less current [40]. In agreement with the electrochemical read-out, the SERS experiments recorded higher Raman signals for experiments with $100 \mathrm{pg}$ protein solution than the $50 \mathrm{pg}$ and $10 \mathrm{pg}$. This signal intensities can be explained by the presence of larger amount of SERS nanotags when more target proteins were captured on the surface and labelled with larger amount of SERS nanotags [41]. Similar observations were also recorded for BRAF and PDL-1 protein samples when ran separately with the above mentioned amounts (Figure $3 b, c, e, f)$. 

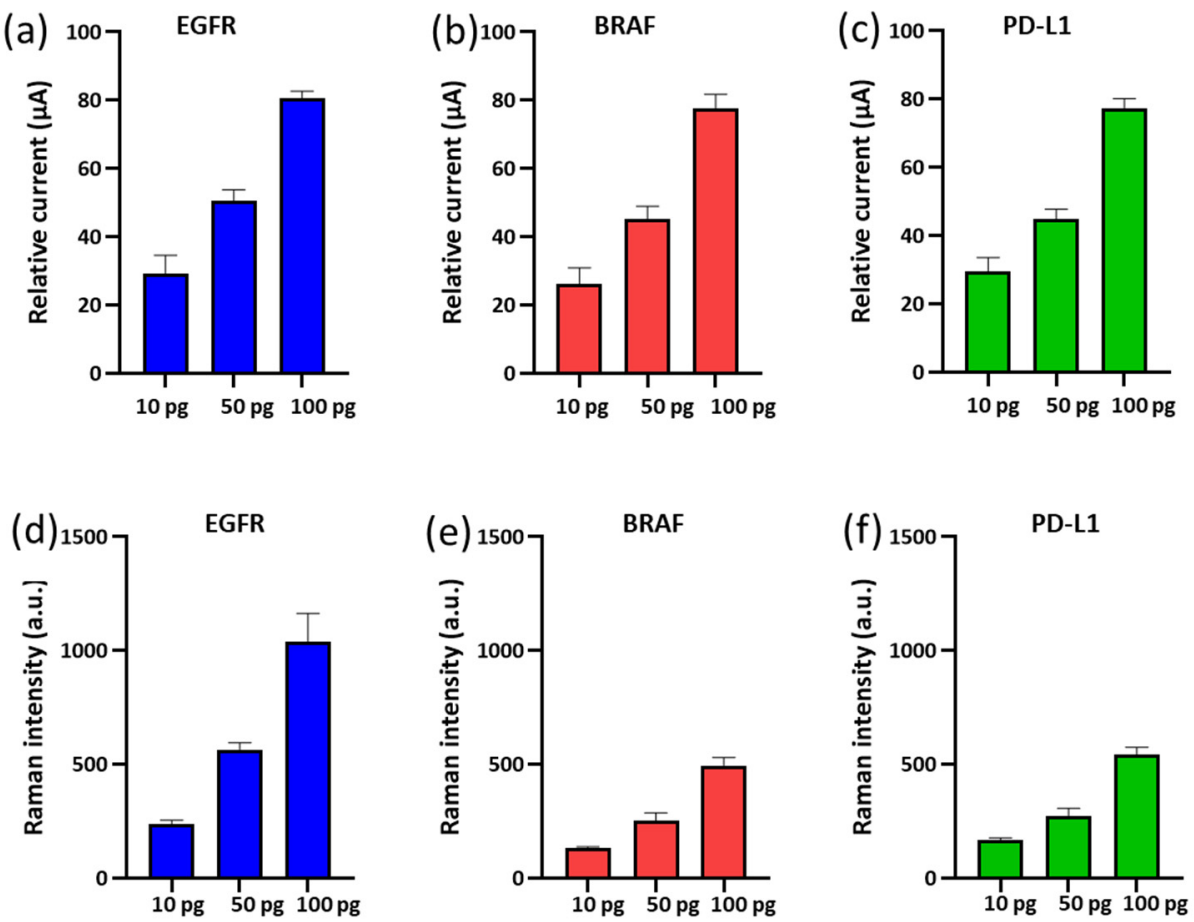

Figure 3. Sensitivity for individual detection of circulating proteins. DPV and SERS measurements of EGFR, BRAF and PDL-1 proteins for three different amounts: $10 \mathrm{pg}, 50 \mathrm{pg}$ and $100 \mathrm{pg}(1 \mathrm{ng} / \mathrm{mL}$, $5 \mathrm{ng} / \mathrm{mL}$ and $10 \mathrm{ng} / \mathrm{mL}$, respectively). (a-c) relative DPV current reduction as a function of increase protein amount from 10-100 ng and (d-f) corresponding average Raman intensities. $(\mathrm{n}=3)$ The Raman signal peak positions for measuring EGFR, BRAF and PDL-1 are $1335 \mathrm{~cm}^{-1}, 1080 \mathrm{~cm}^{-1}$ and $1376 \mathrm{~cm}^{-1}$, respectively. The error bars represent the standard deviation from three replicate runs.

\subsection{Ensemble and Individual Detection of a Mixture of Circulating EGFR, BRAF and PDL-1}

Finally, we performed experiments with three different mixtures of target protein solutions for both ensemble protein analysis with DPV and individual protein analysis with SERS. For the analysis of mixed population, three different samples were prepared where the total circulating protein concentration was $70 \mathrm{pg}$. Sample 1 contained $50 \mathrm{pg}$ of BRAF, $10 \mathrm{pg}$ of EGFR and $10 \mathrm{pg}$ of PDL-1. Sample 2 contained $10 \mathrm{pg}$ BRAF, $10 \mathrm{pg}$ EGFR and 50 pg PDL-1. Sample 3 contained 10 pg BRAF, 50 pg EGFR and 10 pg PDL-1. The sample volume was kept constant at $10 \mu \mathrm{L}$. As the total amount of protein remained constant (i.e., $70 \mathrm{pg}$ ), we expected to observe a similar DPV signal change for the three tested samples. Indeed, the DPV signals for all the samples showed similar current change $(\sim 60 \%)$ (Figure $4 a)$. Figure 4 b,c shows a representative example for the analysis of sample 1 with ensemble protein analysis by DPV and individual protein analysis by SERS. These findings also highlight the essentiality of having a dual readout method for heterogenous sample analysis as similar current changes were recorded for all three samples although each sample contained different amount of individual proteins. Further analysis of each samples using SERS spectra clearly mapped individual protein expressions in samples 1-3. Results of EGFR expression in all three samples are presented in Figure $4 d$, where SERS intensity obtained for EGFR protein detection for sample 3 was similar to the intensity for $50 \mathrm{pg}$ of EGFR protein recorded in Figure 3d and for other two samples, SERS intensities for EGFR was similar to the intensity recorded for $10 \mathrm{pg}$ in Figure 3d. Similarly, in Figure 4e,f BRAF and PDL-1 signals for sample 1 and 2 also showed matching SERS signals for BRAF (50 pg) and PDL-1 (50 pg) in Figure 3e,f, respectively. SERS signals for BRAF protein in sample 2 and 3 were similar to SERS signal of $10 \mathrm{pg}(1 \mathrm{ng} / \mathrm{mL})$ when detected separately (Figure 3e). Low SERS signals were also detected for PDL-1 in sample 1 and 3 as expected due to their low amount (10 pg) in these two samples. (Figure $4 \mathrm{f}$ ) A heatmap in Figure $4 \mathrm{~g}$ presents SERS intensity profile of all three proteins in each sample where grey colour 
represents low SERS signal correspond to less target protein where red colour represent strong SERS signal that represents to more protein capture. (Figure $4 \mathrm{~g}$ ) SERS images of all three samples also reflects the presence of different protein amounts in each samples depicted by three different coloured dots (red = BRAF, green $=$ EGFR, and blue $=$ PDL-1) (Figure $4 \mathrm{~h}$ ). This results clearly demonstrate the feasibility of the current assay platform for ensemble and individual circulating biomarkers analysis in the same sample.
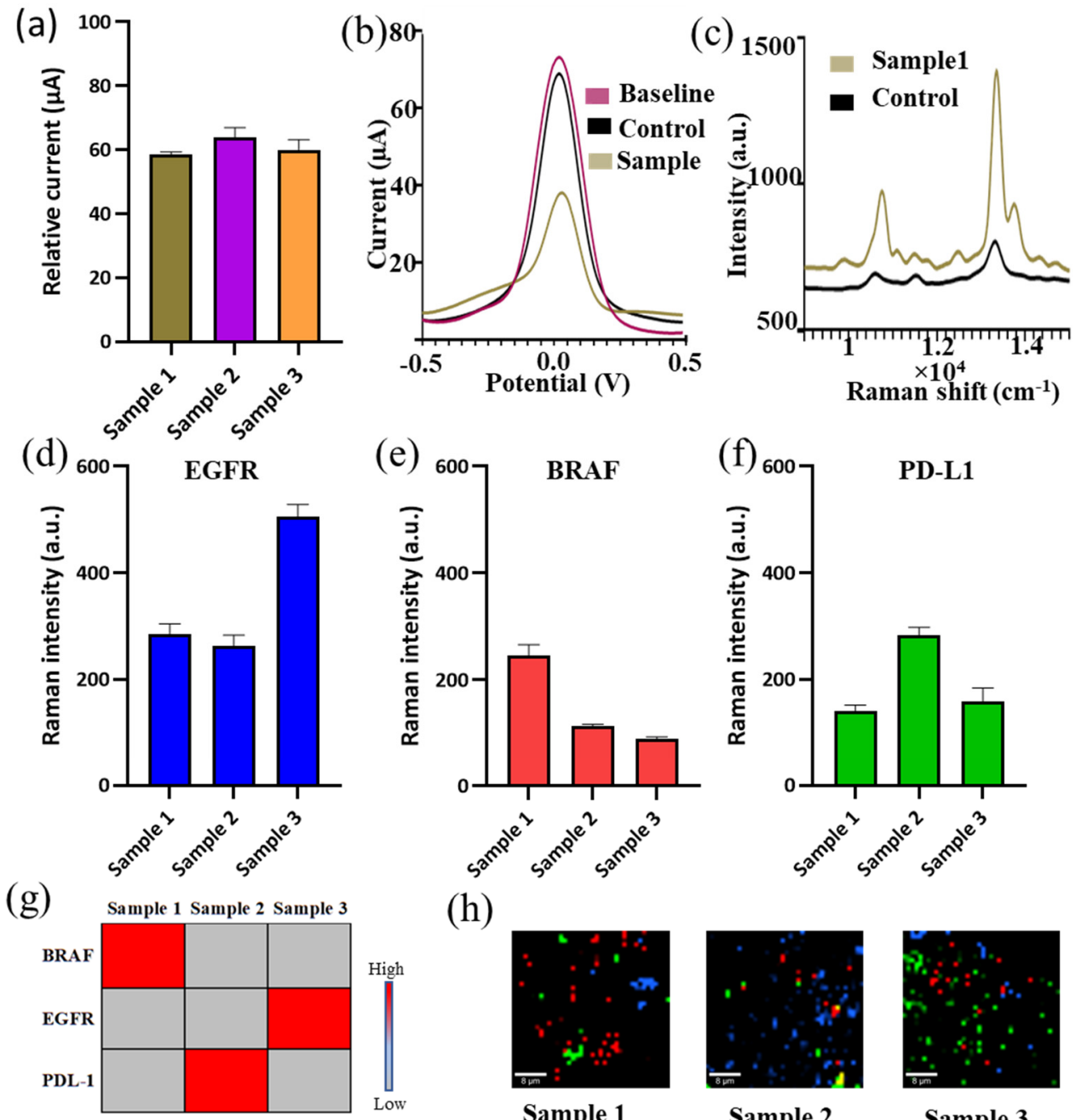

(h)

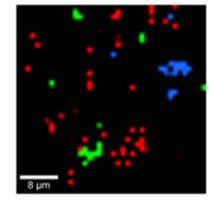

Sample 1

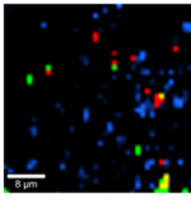

Sample 2

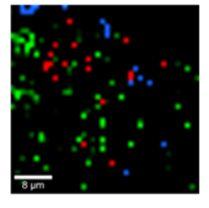

Sample 3

Figure 4. Ensemble and individual detection of mixtures of BRAF, EGFR, and PDL-1. (a) represents the DPV signals of ensemble measurements of samples 1 to $3 ;(\mathbf{b}, \mathbf{c})$ represents the DPV measurement and corresponding SERS spectra of sample 1. (d-f) Average SERS intensities of three biomarkers analysed. The error bars represent the standard errors from three replicate runs. (g) Heatmap of SERS intensities for three proteins in each sample $(\mathbf{h})$ False colour SERS images (Red $=$ BRAF, green $=$ EGFR and blue $=$ PDL-1) of sample 1 , sample 2 and sample 3 , respectively.

\section{Discussion}

Soluble proteins are critical for cancer progression and offer potential cargo of information for disease diagnosis and prognosis [42,43]. Analysis of multiple clinically relevant proteins is considered as the core of interest for developing biosensing platforms. However, detecting low abundance biomarkers within a complex mixture of other biomolecules in biological fluids aggravates the associated challenges of successfully assaying these protein biomarkers [44]. In this work, we develop a proof-of-concept method to overcome some of the key challenges for circulating biomolecular analysis while offering a dual mode of detection to improve assay performance and potentially improve diagnostics. The dual 
detection biosensor is demonstrated for simultaneously analysing ensemble and individual cancer-associated, circulating biomarkers as low as $10 \mathrm{pg}$ per biomarker. Although three different protein concentrations are investigated in this study, evaluation of the quantitative range of the method with more protein concentrations should be performed to enable quantification of protein biomarkers. Our method uses ac-EHD induced nanomixng and microelectrode surface blocking to reduce non-target sensor binding, which we believe is promising to analyse clinical samples (e.g., plasma). The unique ability to process and analyse multiple biomarkers within the same sensing zone of this biosensor could offer more control in biomolecular analysis in terms of minimal sample requirement, quick analysis and dual readouts of the same sample that may improve assay quality.

\section{Conclusions}

In this report, we develop a dual detection biosensor platform that employs electrochemical, and Raman based readouts for screening of the presence of cancer-associated, circulating protein biomarkers and sensitive individual biomarkers profiling. Rapid cancer screening is facilitated by DPV for detecting ensemble protein biomarkers captured on sensor surface and subsequently SERS readout enables to precisely profile each type of the circulating biomarker captured on the surface. Our method is sensitive and can map multiple biomarkers in a spiked patient sample with their individual contribution of as low as $10 \mathrm{pg}$. Although the method is demonstrated for three biomarkers, we believe the multiplexing capability can be further increased by employing a larger panel of antibodies and SERS nanotags. We envisage, this novel dual detection biosensor platform with more validation could offer a better diagnosis for sample analysis which may significantly improve cancer management and treatment outcome.

Supplementary Materials: The following supporting information can be downloaded at: https: / / www.mdpi.com/article/10.3390/chemosensors10030093/s1, Figure S1: Device Fabrication; Figure S2: Raman signals of SERS nanotags; Figure S3: DPV signals during biosensor functionalization. Figure S4: DPV signals during biosensor functionalization. Successful immobilization of mole-cules on the biosensor surface is confirmed by current reduction as bare Au (gold) > DSP func-tionalization $>$ Antibody functionalization > MCH blocking (baseline for target biomolecule anal-ysis).

Author Contributions: Conceptualization, S.D., A.A.I.S., A.W. and M.T.; methodology, S.D., E.A. and P.S.S.; formal analysis, S.D., E.A., P.S.S., A.A.I.S., A.W. and M.T.; investigation, S.D., E.A., P.S.S., A.A.I.S., A.W. and M.T.; data curation, S.D., E.A. and P.S.S.; writing-original draft preparation, E.A., S.D., P.S.S., A.A.I.S., A.W. and M.T.; writing-review and editing, S.D., A.A.I.S., A.W. and M.T.; visualization, S.D., E.A., A.A.I.S. and A.W.; supervision, S.D., A.A.I.S., A.W. and M.T.; project administration, S.D., A.A.I.S., A.W. and M.T.; funding acquisition, A.A.I.S., A.W. and M.T. All authors have read and agreed to the published version of the manuscript.

Funding: This research was funded by the Australian Research Council (DP210103151, DE200100345), National Health and Medical Research Council (APP1175047 and APP1173669), and Cancer Australia (ID2010799).

Data Availability Statement: The data presented in this study are available in the article or Supporting Information.

Acknowledgments: The authors thank the Australian National Fabrication Facility, Queensland Node, for support with device fabrication.

Conflicts of Interest: The authors declare no conflict of interest.

\section{References}

1. Fisher, R.; Pusztai, L.; Swanton, C. Cancer heterogeneity: Implications for targeted therapeutics. Br. J. Cancer 2013, 108, 479-485. [CrossRef] [PubMed]

2. Palmirotta, R.; Lovero, D.; Cafforio, P.; Felici, C.; Mannavola, F.; Pellè, E.; Quaresmini, D.; Tucci, M.; Silvestris, F. Liquid biopsy of cancer: A multimodal diagnostic tool in clinical oncology. Ther. Adv. Med. Oncol. 2018, 10, 1758835918794630. [CrossRef]

3. Alix-Panabieres, C. The future of liquid biopsy. Nature 2020, 579, S9. [CrossRef] [PubMed] 
4. Pantel, K.; Alix-Panabières, C. Real-time liquid biopsy in cancer patients: Fact or fiction? Cancer Res. 2013, 73, 6384-6388. [CrossRef] [PubMed]

5. Hofman, P.; Heeke, S.; Alix-Panabières, C.; Pantel, K. Liquid biopsy in the era of immuno-oncology: Is it ready for prime-time use for cancer patients? Ann. Oncol. 2019, 30, 1448-1459. [CrossRef]

6. $\quad$ Birkenkamp-Demtröder, K.; Christensen, E.; Nordentoft, I.; Knudsen, M.; Taber, A.; Høyer, S.; Lamy, P.; Agerbæk, M.; Jensen, J.B.; Dyrskjøt, L. Monitoring treatment response and metastatic relapse in advanced bladder cancer by liquid biopsy analysis. Eur. Urol. 2018, 73, 535-540. [CrossRef]

7. Zhu, X.; Lang, J. Soluble PD-1 and PD-L1: Predictive and prognostic significance in cancer. Oncotarget 2017, 8, 97671. [CrossRef]

8. Baron, A.T.; Wilken, J.A.; Haggstrom, D.E.; Goodrich, S.T.; Maihle, N.J. Clinical implementation of soluble EGFR (sEGFR) as a theragnostic serum biomarker of breast, lung and ovarian cancer. IDrugs 2009, 12, 302-308.

9. Chen, Y.; Wang, Q.; Shi, B.; Xu, P.; Hu, Z.; Bai, L.; Zhang, X. Development of a sandwich ELISA for evaluating soluble PD-L1 (CD274) in human sera of different ages as well as supernatants of PD-L1+ cell lines. Cytokine 2011, 56, 231-238. [CrossRef]

10. Chu, B.; Qi, T.; Liao, J.; Peng, J.; Li, W.; Fu, S.; Luo, F.; Qian, Z. Colorimetric detection of cancer biomarker based on pH induced color change. Sens. Actuators B Chem. 2012, 166, 56-60. [CrossRef]

11. Wegner, K.D.; Lindén, S.; Jin, Z.; Jennings, T.L.; el Khoulati, R.; van Bergen en Henegouwen, P.M.; Hildebrandt, N. Nanobodies and nanocrystals: Highly sensitive quantum dot-based homogeneous FRET immunoassay for serum-based EGFR detection. Small 2014, 10, 734-740. [CrossRef]

12. Wu, S.; Liu, L.; Li, G.; Jing, F.; Mao, H.; Jin, Q.; Zhai, W.; Zhang, H.; Zhao, J.; Jia, C. Multiplexed detection of lung cancer biomarkers based on quantum dots and microbeads. Talanta 2016, 156, 48-54. [CrossRef] [PubMed]

13. Qu, F.; Li, T.; Yang, M. Colorimetric platform for visual detection of cancer biomarker based on intrinsic peroxidase activity of graphene oxide. Biosens. Bioelectron. 2011, 26, 3927-3931. [CrossRef] [PubMed]

14. Antal-Szalmás, P.; Nagy, B., Jr.; Debreceni, I.B.; Kappelmayer, J. Measurement of soluble biomarkers by flow cytometry. EJIFCC 2013, 23, 135. [PubMed]

15. Nimse, S.B.; Sonawane, M.D.; Song, K.-S.; Kim, T. Biomarker detection technologies and future directions. Analyst 2016, 141, 740-755. [CrossRef]

16. Chikkaveeraiah, B.V.; Bhirde, A.A.; Morgan, N.Y.; Eden, H.S.; Chen, X. Electrochemical immunosensors for detection of cancer protein biomarkers. ACS Nano 2012, 6, 6546-6561. [CrossRef]

17. Díaz-Fernández, A.; Lorenzo-Gómez, R.; Miranda-Castro, R.; de-Los-Santos-Álvarez, N.; Lobo-Castañón, M.J. Electrochemical aptasensors for cancer diagnosis in biological fluids-A review. Anal. Chim. Acta 2020, 1124, 1-19. [CrossRef]

18. Hasan, M.; Ahommed, M.; Daizy, M.; Bacchu, M.; Ali, M.; Al-Mamun, M.; Aly, M.A.S.; Khan, M.; Hossain, S. Recent development in electrochemical biosensors for cancer biomarkers detection. Biosens. Bioelectron. X 2021, 8, 100075. [CrossRef]

19. Ilkhani, H.; Sarparast, M.; Noori, A.; Bathaie, S.Z.; Mousavi, M.F. Electrochemical aptamer/antibody based sandwich immunosensor for the detection of EGFR, a cancer biomarker, using gold nanoparticles as a signaling probe. Biosens. Bioelectron. 2015, 74, 491-497. [CrossRef]

20. Moon, J.-M.; Kim, Y.H.; Cho, Y. A nanowire-based label-free immunosensor: Direct incorporation of a PSA antibody in electropolymerized polypyrrole. Biosens. Bioelectron. 2014, 57, 157-161. [CrossRef]

21. Bravo, K.; Ortega, F.G.; Messina, G.A.; Sanz, M.I.; Fernández-Baldo, M.A.; Raba, J. Integrated bio-affinity nano-platform into a microfluidic immunosensor based on monoclonal bispecific trifunctional antibodies for the electrochemical determination of epithelial cancer biomarker. Clin. Chim. Acta 2017, 464, 64-71. [CrossRef]

22. RáGonšales, V.; JustináGooding, J. Ultrasensitive detection of programmed death-ligand 1 (PD-L1) in whole blood using dispersible electrodes. Chem. Commun. 2021, 57, 2559-2562.

23. Dey, S.; Koo, K.M.; Wang, Z.; Sina, A.A.; Wuethrich, A.; Trau, M. An integrated multi-molecular sensor for simultaneous BRAF V600E protein and DNA single point mutation detection in circulating tumour cells. Lab Chip 2019, 19, 738-748. [CrossRef] [PubMed]

24. Guerrini, L.; Alvarez-Puebla, R.A. Surface-enhanced Raman spectroscopy in cancer diagnosis, prognosis and monitoring. Cancers 2019, 11, 748. [CrossRef]

25. Vendrell, M.; Maiti, K.K.; Dhaliwal, K.; Chang, Y.-T. Surface-enhanced Raman scattering in cancer detection and imaging. Trends Biotechnol. 2013, 31, 249-257. [CrossRef]

26. Granger, J.H.; Granger, M.C.; Firpo, M.A.; Mulvihill, S.J.; Porter, M.D. Toward development of a surface-enhanced Raman scattering (SERS)-based cancer diagnostic immunoassay panel. Analyst 2013, 138, 410-416. [CrossRef] [PubMed]

27. Dey, S.; Trau, M.; Koo, K.M. Surface-enhanced raman spectroscopy for cancer immunotherapy applications: Opportunities, challenges, and current progress in nanomaterial strategies. Nanomaterials 2020, 10, 1145. [CrossRef] [PubMed]

28. Wang, Y.; Vaidyanathan, R.; Shiddiky, M.J.; Trau, M. Enabling rapid and specific surface-enhanced Raman scattering immunoassay using nanoscaled surface shear forces. ACS Nano 2015, 9, 6354-6362. [CrossRef] [PubMed]

29. Li, J.; Wuethrich, A.; Sina, A.A.; Cheng, H.-H.; Wang, Y.; Behren, A.; Mainwaring, P.N.; Trau, M. A digital single-molecule nanopillar SERS platform for predicting and monitoring immune toxicities in immunotherapy. Nat. Commun. 2021, $12,1087$. [CrossRef]

30. Yang, H.; Gun, X.; Pang, G.; Zheng, Z.; Li, C.; Yang, C.; Wang, M.; Xu, K. Femtosecond laser patterned superhydrophobic/hydrophobic SERS sensors for rapid positioning ultratrace detection. Opt. Express 2021, 29, 16904-16913. [CrossRef] 
31. Xu, L.; Liu, H.; Zhou, H.; Hong, M. One-step fabrication of metal nanoparticles on polymer film by femtosecond LIPAA method for SERS detection. Talanta 2021, 228, 122204. [CrossRef] [PubMed]

32. Kozhina, E.; Andreev, S.; Tarakanov, V.; Bedin, S.; Doludenko, I.; Naumov, A. Study of local fields of dendrite nanostructures in hot spots formed on SERS-active substrates produced via template-assisted synthesis. Bull. Russ. Acad. Sci. Phys. 2020, 84, 1465-1468. [CrossRef]

33. Kozhina, E.P.; Bedin, S.A.; Nechaeva, N.L.; Podoynitsyn, S.N.; Tarakanov, V.P.; Andreev, S.N.; Grigoriev, Y.V.; Naumov, A.V. Ag-nanowire bundles with gap hot spots synthesized in track-etched membranes as effective SERS-substrates. Appl. Sci. 2021, 11, 1375. [CrossRef]

34. Shiddiky, M.J.; Vaidyanathan, R.; Rauf, S.; Tay, Z.; Trau, M. Molecular nanoshearing: An innovative approach to shear off molecules with AC-induced nanoscopic fluid flow. Sci. Rep. 2014, 4, 3716. [CrossRef] [PubMed]

35. Frens, G. Controlled nucleation for the regulation of the particle size in monodisperse gold suspensions. Nat. Phys. Sci. 1973, 241, 20-22. [CrossRef]

36. Zorea, J.; Shukla, R.P.; Elkabets, M.; Ben-Yoav, H. Probing antibody surface density and analyte antigen incubation time as dominant parameters influencing the antibody-antigen recognition events of a non-faradaic and diffusion-restricted electrochemical immunosensor. Anal. Bioanal. Chem. 2020, 412, 1709-1717. [CrossRef]

37. Kneipp, K.; Kneipp, H.; Kneipp, J. Surface-enhanced Raman scattering in local optical fields of silver and gold nanoaggregates from single-molecule Raman spectroscopy to ultrasensitive probing in live cells. Acc. Chem. Res. 2006, 39, 443-450. [CrossRef]

38. Pérez-Jiménez, A.I.; Lyu, D.; Lu, Z.; Liu, G.; Ren, B. Surface-enhanced Raman spectroscopy: Benefits, trade-offs and future developments. Chem. Sci. 2020, 11, 4563-4577. [CrossRef]

39. Hong, S.; Li, X. Optimal size of gold nanoparticles for surface-enhanced Raman spectroscopy under different conditions. $J$. Nanomater. 2013, 2013. [CrossRef]

40. Chen, Z.; Liu, X.; Liu, D.; Li, F.; Wang, L.; Liu, S. Ultrasensitive electrochemical DNA biosensor fabrication by coupling an integral multifunctional zirconia-reduced graphene oxide-thionine nanocomposite and exonuclease I-assisted cleavage. Front. Chem. 2020, 8, 521. [CrossRef]

41. Shaw, C.P.; Fan, M.; Lane, C.; Barry, G.; Jirasek, A.I.; Brolo, A.G. Statistical correlation between SERS intensity and nanoparticle cluster size. J. Phys. Chem. C 2013, 117, 16596-16605. [CrossRef]

42. Zhou, Q.; Andersson, R.; Hu, D.; Bauden, M.; Kristl, T.; Sasor, A.; Pawłowski, K.; Pla, I.; Hilmersson, K.S.; Zhou, M. Quantitative proteomics identifies brain acid soluble protein 1 (BASP1) as a prognostic biomarker candidate in pancreatic cancer tissue. EBioMedicine 2019, 43, 282-294. [CrossRef] [PubMed]

43. Welsh, J.B.; Sapinoso, L.M.; Kern, S.G.; Brown, D.A.; Liu, T.; Bauskin, A.R.; Ward, R.L.; Hawkins, N.J.; Quinn, D.I.; Russell, P.J. Large-scale delineation of secreted protein biomarkers overexpressed in cancer tissue and serum. Proc. Natl. Acad. Sci. USA 2003, 100, 3410-3415. [CrossRef] [PubMed]

44. Ray, S.; Reddy, P.J.; Jain, R.; Gollapalli, K.; Moiyadi, A.; Srivastava, S. Proteomic technologies for the identification of disease biomarkers in serum: Advances and challenges ahead. Proteomics 2011, 11, 2139-2161. [CrossRef] 